\title{
BROAD ABSORPTION LINE QSOs
}

\author{
David A. Turnshek \\ Space Telescope Science Institute \\ Baltimore, MD, 21218 \\ USA
}

\begin{abstract}
Observational constraints on models for broad absorption line (BAL) QSOs are discussed. The picture which emerges is one in which the broad absorption line region (BALR) contributes to the broad emission line region (ELR) as a high ionization component. Depending on QSO luminosity, the limits on the distance of the BALR from the central source are 1 - 1000 pc. A highly metal enriched and disk-like geometry for the region is indicated. The similarity between the absorption in some radio quiet BAL QSOs and the associated complexes of absorption in some radio loud QSOs may indicate that the BALR outflow can affect the region surrounding a QSO out to distances in excess of several hundred kpc.
\end{abstract}

\section{Introduction}

Several review-type papers dealing exclusively with BAL QSOs have appeared recently (Weymann and Foltz 1983; Turnshek 1984a; Weymann, Turnshek and Christiansen 1985). As a result, this review paper makes only a brief summary of past observations of BAL QSOs, but it does try to put them into perspective. Newer observations are reviewed in more detail. In particular, I discuss recent observations which pertain to the fraction of BAL QSOs as a function of redshift, observations concerning the ELs of BAL QSOs, the derivation of column densities of BAL clouds which are relevant to abundance determinations and observations which constrain possible changes in BAL profiles with time. I discuss in more detail how the observations are capable of placing constraints on models of QSOs and BALRs. Imposed constraints have implications for geometry, size scales and abundances. I also review some interesting observations of radio loud QSOs with associated complex absorption and discuss how this absorption may be related to the BAL phenomenon. Finally, I discuss problems and the needs for future work.

\section{Review of Observations}

\subsection{General Characteristics}

A BAL QSO shows a substantial amount of resonance line absorption blueshifted with respect to the emission redshift. I suspect that at the present time over 100 BAL QSOs may be known to various observers, but only half that number are 
suggested from data in the literature. A small fraction of these could be considered well-studied. Ninety-five percent of the time, the absorption has only a high level of ionization and transitions of CIV $\lambda 1549, \mathrm{NV} \lambda 1240$ and OVI $\lambda 1034$ are dominant; $\operatorname{SiIV} \lambda 1397$ is moderate and $\mathrm{Ly}-\alpha$ is moderate to weak. Five percent of the time, the absorption also has low ionization components and transitions of $\mathrm{MgII} \lambda 2798$ and sometimes CII $\lambda 1335$ are present. Other reported weak to intermediate strength transitions include AlIII $\lambda 1857$ and CIII $\lambda$ 977. There is less information on the frequency of appearance of these other transitions because they fall in wavelength regions not always observed, but they do appear more often than MgII and CII. Although hydrogen Lyman limit absorption has never been observed, BALs have been detected in the Lyman continuum (e.g. see Alec Boksenberg's contribution at this symposium). BALs generally only appear in radio quiet QSOs (Stocke et al. 1984), but see section 4 for some qualifications on this effect. The BAL profiles can have a smooth appearance or a very broken-up appearance, with multiple troughs and/or individual absorption line doublet components present. Very often the absorption is detached with respect to the peak emission redshift. Absorptionabsorption line-locking is sometimes present (e.g. see Foltz et al. 1986a). The absorption outflow velocity typically ranges up to $20,000-30,000 \mathrm{~km} \mathrm{~s}^{-1}$ and in one case it may range up to $60,000 \mathrm{~km} \mathrm{~s}^{-1}$. However, cases where the absorption has an extent of only a few thousand $\mathrm{km} \mathrm{s}^{-1}$ also exist. Multiple troughs are said to be present when several discrete complexes or troughs of absorption exist, separated by several thousand $\mathrm{km} \mathrm{s}^{-1}$. The existence of multiple troughs is significant because of the potential constraints they may impose on model geometry (see section 3.1). In cases where the absorption has an extent of a little less than a few thousand $\mathrm{km} \mathrm{s}^{-1}$, whether the absorption is fundamentally similar to the BALs or whether it is due to a related or completely different phenomenon is unclear (see section 4).

In their review article Weymann and Foltz (1983) present a nice compendium of BAL QSO spectra. For convenience I illustrate two typical types of spectra in Figures 1 and 2. The wavelength scales in the Figures have been shifted into

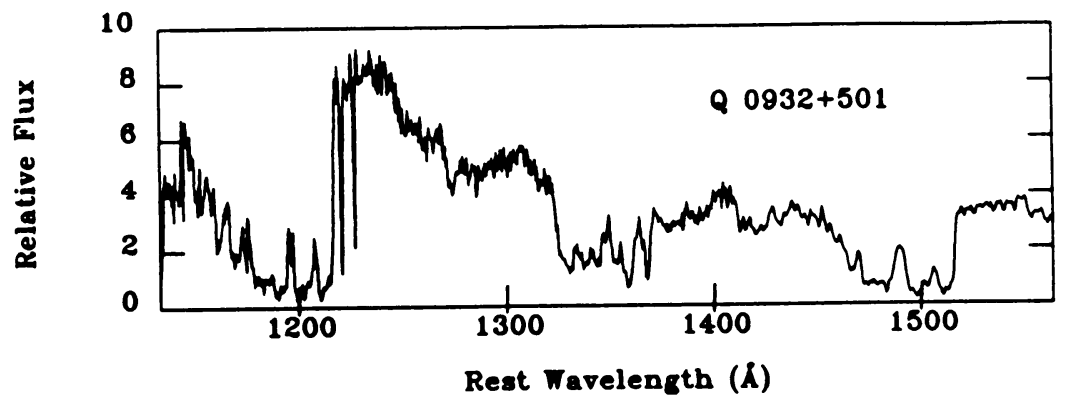

Fig. 1: BAL QSO exhibiting complex absorption component structure.

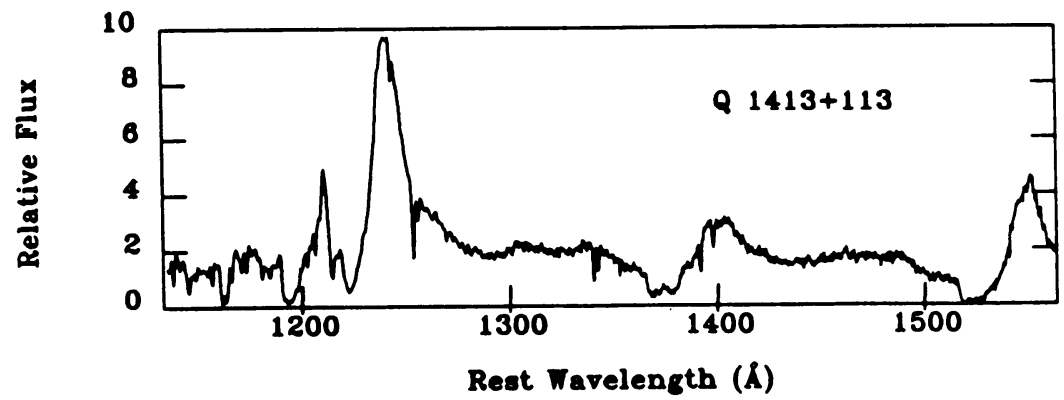

Fig. 2: BAL QSO exhibiting smooth absorption. 
the QSO restframe. As opposed to some of the most complex BAL QSO spectra (e.g. Q1414+087 in Foltz et al. 1983a and UM141 in Turnshek et al. 1980), these spectra are fairly easy to assess because outflow velocities are not high enough to cause much confusion due to overlapping transitions from different ionic species. The object in Figure 1 has detached absorption showing considerable structure in the trough, whereas the object in Figure 2 shows considerably smoother absorption which may also be somewhat detached. See Turnshek (1984a) for a discussion of how the combination of optical depth effects, cloud sizes and kinematics can influence the observed characteristics of BAL profiles.

BAL QSOs are also the only class of QSOs which are radio quiet and can have a polarization in excess of a few percent. Finally, a small amount of data suggests that BAL QSOs are more optically variable than their radio quiet non-BAL QSO counterparts. See Turnshek (1984a) for more details about these two effects.

\subsection{Fraction of QSOs with BALs as a Function of Redshift}

Hazard et al. (1984) has estimated that BAL QSOs comprise $0.03-0.10$ of the QSO population near redshifts of 2.3; there is no evidence that this fraction varies significantly with redshift. I emphasize this latter point to an even greater extent than I did in my talk because shortly after this symposium, a search of the IUE archives for other purposes resulted in the discovery of two new low redshift BAL QSOs (Turnshek and Grillmair 1986). Both of these objects are identified in the Palomar-Green Bright Quasar Survey (BQS). In addition, Matt Malkan reported at this conference (see the discussion following this paper) that during the course of his IUE observations, he recently discovered a very low redshift BAL QSO identified in the BQS. Coupled with previous identifications of BAL QSOs in the BQS (Wilkes 1985; Turnshek et al. 1985), this means that there are at least 5 low redshift BAL QSOs in the BQS sample. I estimate that only about 50 objects identified in the BQS have been observed in sufficient detail to have discovered BALs. The mean redshift of the five known BQS BAL QSOs is about 0.5. Hence, the fraction of QSOs with BALs at low redshift is compatible with the $0.03-0.10$ fraction given by Hazard et al. (1984). However, Cyril Hazard (private communication) has noted that in his survey work he finds a greater tendency for BALs to be present in the spectra of the highest redshift QSOs. Since selection effects are so controversial and hard to evaluate at the highest redshifts, more work will have to be done to explore this important possibility.

\subsection{Emission Lines of BAL QSOs}

One interesting statistical observation concerning BAL QSOs is that the distribution of their emission line (EL) properties differs from those of non-BAL QSOs (Turnshek 1984a; Hartig and Baldwin 1986). For instance, after the effects of absorption are taken into account, CIV emission tends to be weaker in BAL QSOs and, although Hartig and Baldwin do not concur, I have said that NV emission is stronger in BAL QSOs. BAL QSOs also appear to have an excess of AlIII and iron emission which complicates the CIII] spectral region. I emphasize that these results on differences between the ELs of BAL QSOs versus non-BAL QSOs refer to differences in the distribution of EL properties between the two classes. For example, for a particular BAL QSO with weak CIV emission, one is likely to have little trouble finding a non-BAL QSO with even weaker CIV emission, but this does not negate the fact that weak CIV emission is a more common property of 
BAL QSOs than of non-BAL QSOs. ${ }^{1}$ Apparently, Turnshek (1984a) and Hartig and Baldwin (1986) reached different conclusions concerning NV emission in BAL QSOs versus non-BAL QSOs simply because the Hartig and Baldwin (1986) nonBAL QSO comparison sample had no objects with very weak NV emission. Since the detailed resolution of the NV question depends so much on understanding selection effects, I believe that the best clarification which can be made at this time is to note that there are non-BAL QSOs which show practically no evidence for NV broad emission (e.g. see Foltz et al. 1983b), while the same is not true for BAL QSOs.

In addition, Hartig and Baldwin (1986) have presented observations of the BAL QSO Q0335-336, a very unusual object which exhibits unusually narrow emission $\left(1000-2000 \mathrm{~km} \mathrm{~s}^{-1}\right)$ and components of FeIII emission near or in place of the CIII] $\lambda 1909 \mathrm{EL}$. This object also has strong FeII emission and an emission feature near $2080 \AA$ that is probably FeII. Non-BAL QSO counterparts to this object have not been discovered to date.

There also appear to be correlations between a QSO's BAL profile type and the corresponding characteristics of the adjacent EL. For example, when comparisons of BAL QSOs are made, one finds that as BAL profiles become less smooth and more detached from the emission, showing multiple troughs and sometimes narrow component structure, the peak emission line height to continuum ratio is generally smaller and the emission line width is usually broader. There may also be a tendency for the characteristic width of the BALs to match the width of the ELs. These general effects can be seen by comparing Figure 1 and Figure 2.

Clearly the systematics of QSO EL and BAL properties are very intricate and large sample sizes are needed to explore the types of systematics I have noted above. This is particularly obvious when one considers that there are many different types of BAL profiles to be explored and yet BAL QSOs comprise only $0.03-0.10$ of the QSO population.

\subsection{Detailed Studies of BAL Profiles}

One of the more interesting aspects of studing BAL QSOs is the determination of column densities and comparison of these column densities to predictions of models for the level of ionization in the BALR. In certain circumstances column densities can be determined fairly accurately (Turnshek 1984a). One object $(\mathrm{Q} 0932+501)$ that I have studied with Frank Briggs, Craig Foltz and Ray Weymann (Turnshek et al. 1986) is worth mentioning because it appears to have a region where the $\mathrm{C}^{+}$column density is only a factor of 2 or so less than the $\mathrm{H}^{0}$ column density. Explaining this observation is difficult unless abundances in the BALR are significantly enhanced relative to solar values (section 3.3).

Foltz et al. (1986a) recently reported on the comparison of two sets of observations of Q1303+308 separated by two years. As Q1303+308 has considerable absorption structure, they were able to put a $3 \sigma$ upper limit on any change in velocity of the absorption components of $30 \mathrm{~km} \mathrm{~s}^{-1}$. This can be used to put a lower limit on the BALR size scale (section 3.2). Comparison of these observations did show that, at a low level of significance, the intensity of some high velocity smoother BAL features did appear to get weaker during the two year time period.

1 I should point out as an aside that BAL QSOs appear to show no evidence for the Baldwin effect and I believe that this is also true for radio quiet QSOs in general. 


\section{Constraints on Models}

\subsection{BALR Covering Factor and Geometry}

Before I begin reviewing the arguments which constrain BALR covering factor and geometry, I will note two constraints which are dealt with more specifically in section 3.2: (1) that the BALR lies outside the broad Ly- $\alpha$ emitting region and (2) that the BALR can contribute a high ionization component to a QSO's broad EL spectrum.

Comparison of observations with resonance line scattering (RLS) models are capable of placing constraints on the global value of the BALR covering factor. If the global covering factor is too large, RLS produces more emission than is observed in certain parts of the spectrum. The global value of the BALR covering factor is simply the fraction of the QSO covered by BALRs as determined by integrating over all possible lines of sight. This should be distinguished from the local BALR covering factor which is determined by integrating only over lines of sight which differ from the line of sight to the observer by small angles. Generally, the value of the global BALR covering factor is constrained to be less than $0.1-0.2$ (Turnshek et al. 1980; Turnshek 1981; Junkkarinen 1983; Turnshek 1984b). The CIV and NV regions of a spectrum provide the most powerful constraints on the maximum value of the covering factor.

Working in the CIV region has the advantage of being able to make a fairly accurate assessment of the unabsorbed continuum level. For the CIV case, if the BAL clouds are distributed in a spherically symmetric manner or in an axially symmetric disk-like manner, the value of the global BALR covering factor is usually constrained by the amount of emission present in the region $5,000-20,000 \mathrm{~km} \mathrm{~s}^{-1}$ to the red of the CIV emission peak and also by the residual intensity in the trough region on the blue side of the CIV emission peak. However, if the BAL clouds are distributed in a jet or fan pointed toward the observer, the residual intensity in the trough is the only constraining factor. There are some cases where the total equivalent width of the CIV emission is so much smaller than typical BAL equivalent widths that the global covering factor must be small $(<0.1-0.2)$, independent of EL profile or residual intensity considerations.

Working in the NV region has the advantage that the radiation scattered by the NV BALs includes not only the inner continuum radiation, but also the inner broad Ly- $\alpha$ EL (see section 3.2). However, since the inner Ly- $\alpha$ EL is attenuated by the NV BAL, one has to assume a value for the Ly- $\alpha$ EL equivalent width in order to calculate the resulting EL equivalent width of NV RLS radiation as a function of global BALR covering factor. If one assumes reasonable values for the unattenuated Ly- $\alpha$ EL equivalent width, one finds that the observed NV EL equivalent also sets upper limits on the global BALR covering factor of $0.1-0.2$. This is a very powerful constraint in a statistical sense, but its value for any one object is unclear.

The most obvious consequence of the constraints on global BALR covering factor is that many QSOs must have BALRs which are not observable via their absorption signature and, since the constraint is an upper limit, all QSOs might have BALRs. Of course, the idea that the global BALR covering factor is constant in QSOs and that'a QSO either does or does not have a BALR may be unrealistic. In fact, if QSOs form any kind of a continuous sequence of objects, the most practical way to think of the global BALR covering factor parameter is that it varies from 0.0 to 0.2 and it applies to all QSOs.

Mechanisms for the acceleration of BALR material were discussed in detail in 
the Weymann, Turnshek and Christiansen (1985) review article and so they will not be discussed here. However, one point that must be emphasized is that constructing a model for the acceleration where multiple absorption troughs originate from a single ejection episode or region in space is difficult. For example, in the context of the discussion of Weymann, Turnshek and Christiansen (1985), a single trough may result from one's line of sight passing through a main cloud which has been accelerated by ram pressure, followed by smaller ablated 'cloudlets' which have been torn off the main cloud and accelerated to a higher speed. Thus, when observing BAL QSOs with multiple troughs, our line of sight is most probably passing through multiple BALRs, each of which, by itself, is of sufficient extent to have the QSO classified a BAL QSO. Since multiple troughs are observed so frequently (approximately $30-40$ percent of the time), interpreting each trough as arising in a separate BALR suggests that the optical depth to BALRs is about 0.7 - 0.9. This requires a fairly large local BALR covering factor of $0.5-0.6$. To see this more explicitly, define the global BALR covering factor to be $q_{c}$ and the solid angle containg BALR clouds to be $\frac{\Omega}{4 \pi}$. If for simplicity $\frac{\Omega}{4 \pi}$ is assumed to be a single valued quantity, i.e., it does not vary from one QSO to the next, then the value of $\mathrm{q}_{c}$ can be computed as the product of two probabilities:

$$
q_{c}=\frac{\Omega}{4 \pi}\left(1-e^{-\eta}\right)
$$

where $\eta$ is the optical depth of isolated BALRs in space and $\left(1-\mathrm{e}^{-\eta}\right)$ is the value of the local BALR covering factor which is the probability that a QSO will be classified as a BAL QSO if our line of sight lies within the solid angle containing BAL clouds. One can see that if $\frac{\Omega}{4 \pi}$ is unity, then the BALR must be spherically symmetric by definition, but if it is less than unity, then a non-spherically symmetric distribution holds. For the case of a spherically symmetric BALR and the realistic range 0.03 $<\mathrm{q}_{c}<0.20,0.03<\eta<0.22$ follows. From Poisson's distribution, the expected distribution of trough multiplicities for $\eta=0.03$ is computed to be: 98.5 percent single troughs, 1.5 percent double troughs and 0.015 percent triple troughs; for $\eta$ $=0.22$ : 89.3 percent single troughs, 10.0 percent double troughs and 0.7 percent triple troughs. As noted, the percentage of double and triple troughs observed is much higher than this, suggesting that the solid angle containing BALRs might typically be only 5 - 35 percent of $4 \pi$ steradians.

Taken together the evidence for a small global BALR covering factor along with a large local BALR covering factor suggests a non-spherically symmetric BALR geometry. The two simplest possible geometries for the BALR would be disklike and jet- or fan-like. Since jet or fan geometries could not produce symmetric emission, a disk geometry is preferred.

\subsection{Size Scale of the BALR and Emission from the BALR}

There are basically three size scale regimes, corresponding to three different models that have been proposed, that are worth noting: (1) the wind model of Drew and Gidding (1982) and Drew and Boksenberg (1984) in which BALR distance scales are much less than 1 pc (typically $0.01-0.003$ pc), (2) the ELR component model of Turnshek (1984b) and Turnshek et al. (1985) in which BALR distance scales are greater than $1 \mathrm{pc}$, but less than $1 \mathrm{kpc}$ and (3) the interstellar cloud - thermal wind model of Weymann et al. (1982) and Scott et al. (1984) in which BALR distance scales are in excess of $1 \mathrm{kpc}$. I will not go into the detailed attributes of each model, but rather discuss constraints on size scales which tend to place the BALR 1 - 1000 pc from the central source, ruling out models (1) and (3). The constraints are generally luminosity dependent. 
Models with size scales less than about $1 \mathrm{pc}$ have difficulty explaining the deduction that the BALR occults the broad Ly- $\alpha$ emitting region (Turnshek, Foltz and Weymann 1986). The effects of this occultation can be seen in Figure 2; note that the bump in the otherwise smooth NV trough is the result of NV absorption attenuating inner continuum plus Ly- $\alpha$ emission and not just attenuating the continuum as is the case near CIV. Since the region giving rise to the broad Ly- $\alpha$ EL is generally thought to have $n_{e} \approx 10^{11} \mathrm{~cm}^{-3}$ (Canfield and Peutter 1981), the Ly- $\alpha$ broad ELR must generally be no more than $\sim 1$ pc from the central source and the BALR lies beyond this. These limits are constrained even further by the recent observations of Foltz et al. (1986a). Assuming the BALR acceleration is constant, the lack of a change in velocity of any of the absorption components requires that the BALR be at least $7 \mathrm{pc}$ from the central source. This lower limit is derived from luminosity independent considerations, but one should remember that it applies to a QSO with a specific luminosity. The assumption that the BALR acceleration is due to radiation pressure leads to the requirement that the BALR be at least $4 \mathrm{pc}$ from the central source.

Upper limits on the size scale of the BALR are inferred from deductions about the emissivity of BAL clouds. As detailed in Turnshek (1984b), collisional excitation in the BALR is clearly capable of providing a significant amount of observable high ionization broad emission. The two possible caveats to this conclusion concern adopting the appropriate value for the global BALR covering factor and the validity of estimating a lower limit for $n_{e} r^{2}$ in the absence of a working model for the BALR level of ionization (see section 3.3). Since the fraction of QSOs with BALRs is so large, appealing to the first caveat is not a way out of having the BALR contribute to a QSO's broad EL spectrum in some objects, but for QSOs having a very small global BALR covering factor the contribution would be very small. The second caveat is more of a caution than anything else as I believe the techniques used to put a lower limit on $n_{e} \mathrm{r}^{2}$ have been conservative. For $n_{e}<10^{6}-10^{7} \mathrm{~cm}^{-3}$ the strongest EL that would be produced in the BALR would be [OIII] $\lambda 5007$ by a large factor. A lower limit on $n_{e}$ in the BALR then results from the lack of broad [OIII] $\lambda 5007$ emission in QSOs, which in turn can be used to infer an upper limit for the size scale of the BALR. This argument was applied to a specific low redshift BAL QSO (PG1700+518) in Turnshek et al. (1985) and now that other low redshift BAL QSOs are known to exist, the argument is even stronger. In PG1700+518, since the Lyman limit luminosity is relatively small and the effective spectral index between the Lyman limit and the local continuum level ${ }^{2}$ near [OIII] $\lambda 5007$ is so steep, the limits placed on $n_{e}$ versus the global BALR covering factor are not extremely tight. The upper limit derived for the size scale of the BALR in PG1700+518 is 30 pc, but this would scale up to $100-1000 \mathrm{pc}$ in the most luminous BAL QSOs.

Concerning collision excitation giving rise to other ELs, as previously noted, there is the potential for the BALR to produce a significant contribution to the higher ionization lines. However, the fact that the flux detected in the NV BAL troughs are often very small generally demands that no broad Ly- $\alpha$ emission be produced in the BALR. This could either be a temperature effect (Turnshek 1984b) or an abundance effect (section 3.3). Flux that is seen in the NV trough is consistent with being flux from the inner broad Ly- $\alpha$ EL, but attenuated by the NV BALs, or possibly some contribution from the narrow ELR which is unattenuated. Note

2 The local continuum level near [OIII] is high due to the presence of substantial FeII emission (Wampler 1986). 
that the observations seldom demand that the CIV BALR occults the adjacent CIV broad ELR.

One compelling piece of evidence which suggests that different ELs often originate in the same region occurs when EL velocity profiles match. As shown by Wilkes and Carswell (1982) and Gaskell (1982) there are differences between low and high ionization lines. One conclusion which might be drawn from the observations of EL profiles is that when the profile of a high ionization EL matches exactly that of the broad Ly- $\alpha$ EL coming from the inner and denser region, the high ionization EL is also likely to arise in this inner part of the broad ELR which is occulted by the BALR. However, in cases where profiles do not match very well, some high ionization broad emission may be arising in the BALR.

The situation for RLS is different. For global BALR covering factors in the 0.1 - 0.2 range, all of the observed NV broad emission could be accounted for by the BALR. A much smaller, but still noteworthy, fraction of CIV broad emission would be caused by this effect. See Turnshek (1984b) for a discussion of how aspect angle might effect the situation. Aspect angle and radiative transfer effects could offer an explanation for why NV is stronger in BAL QSOs. A general conclusion one might reach from RLS considerations is that a QSO with little or no NV broad emission in its spectrum may have a BALR with very small global covering factor, whereas a QSO with significant NV broad emission in its spectrum may have a much larger global BALR covering factor. The velocity extent of the BALR is also an important parameter in estimating the amount of RLS NV emission.

\subsection{Abundances in the BALR}

Simple models for the level of ionization in the BALR are not very successful at predicting derived column densities. The basic problem which applies to all BAL QSOs is that central source photoionization models adopting solar abundances and assuming either a power law relation for the distribution of ionizing photons or a power law plus blackbody for the distribution of ionizing photons generally fail to produce observed $\mathrm{Si}^{+3}$ column densities without producing much more $\mathrm{H}^{0}$ than is observed (Turnshek 1981; Weymann, Turnshek and Christiansen 1985). When the parameters describing the power law and blackbody contribution to the ionizing photons are adjusted optimally so that the $\mathrm{Si}^{+3}$ to $\mathrm{H}^{0}$ column density ratio is in fair agreement with the observations, the parameterization of the ionizing spectrum is not what one really expects it to be and, additionally, the $\mathrm{C}^{+3}, \mathrm{~N}^{+4}$ and $\mathrm{O}^{+5}$ column densities are factors of $10-1000$ lower than their observed values. Simple collisional ionization models characterized by solar abundances and a small range of temperatures also offer no hope of explaining BALR column densities.

At the present time, one must try to decide whether abundance or model assumptions are lacking. On the one hand, none of the photoionization models that have been calculated take into account the possibility of absorption in the Lyman continuum. This was because the observations demonstrate that BAL clouds are not optically thick at the hydrogen Lyman limit. However, observations in the Lyman continuum of BAL QSOs are now telling us that, although BAL clouds are not optically thick at the Lyman limit, BALs in the Lyman continuum can be present, and this will alter the effective ionizing spectrum that the BALR sees. Any new work utilizing photoionization models for the BALR should try to account for absorption in the Lyman continuum by BALs. Also, there are conceivably many other sources of energy input for such an energetic outflow. Furthermore, adjusting the abundances is not too attractive because the $\mathrm{Si}, \mathrm{C}, \mathrm{N}$ and $\mathrm{O}$ all required different factors. 
On the other hand, based on the observation discussed in section 2.4 , I believe the situation should now be viewed from a different perspective when attempting to assess whether abundance assumptions or model assumptions are lacking. In particular, any model for the level of ionization of BALR clouds will have difficulty explaining an $\mathrm{H}^{0}$ column density only a factor of 2 or so larger than the $\mathrm{C}^{+}$column density without having carbon abundances enhanced 10 - 100 times solar values. Observations of FeII emission in the BAL QSO PG1700+518 (Wampler 1986) may be telling us that iron is similarly enhanced.

\section{Radio Properties of QSOs with Absorption}

BAL QSOs are known to be radio quiet. However, a number of radio loud QSOs having extensive associated complexes of absorption come close to being included in the BAL class. The point is, while these associated complexes of absorption are not as extensive as the BAL absorption, the absorption is extensive enough to raise questions about whether the associated complexes of absorption can be explained by so-called intervening material, even if the material is taken to reside in clusters (Morris et al. 1986). Briggs, Turnshek and Wolfe (1984) have discussed the situation and possible implications. In particular, these authors point out that past work on the so-called marginal BAL QSO PKS1157+014 was somewhat misleading in that broad $\mathrm{Ly}-\alpha$ absorption was taken to have a BAL nature. In fact, the broad Ly- $\alpha$ absorption is due to a damped Ly- $\alpha$ line in the wing of Ly$\alpha$ emission. Systems characterized by damped Ly- $\alpha$ are most easily explained by intervening galaxy disks (Wolfe et al. 1986). However, even if the damped Ly$\alpha$ absorption is excluded from consideration, the absorption in PKS1157+014 is still quite extensive. Similarly, Morris et al. (1986) have analyzed the spectrum of the radio source GC1556+335 and have shown that, although Weymann et al. (1979) initially considered this object to be a possible member of the BAL class, the absorption is not quite extensive enough and the physical conditions in the GC1556+335 absorbing clouds are not the same as in the BAL clouds. Finally, a survey by Foltz et al. (1986b) has shown that $\mathrm{z}_{a b s}$ near $\mathrm{z}_{\text {em }}$ systems, which are similar to the associated complexes of absorption, are generally found in radio loud QSOs, but not in radio quiet QSOs.

The main result of the above referenced work is that the properties of the clouds producing the BALs in radio quiet QSOs are quite different from the properties of the clouds producing the associated complexes of absorption in radio loud QSOs. There are kinematic, level of ionization and abundance differences. In addition, as Morris et al. (1986) discuss, from considerations of CII and CII f.s. one finds that the clouds responsible for the associated complexes of absorption in GC1556+335 are at least several hundred $\mathrm{kpc}$ from the central QSO. Yet, the similarity between the spectra of some BAL QSOs with absorption component structure and the spectra of some radio loud QSOs with associated complexes of absorption gives one the impression that there may be continuity between the absorption line morphology of the two classes of objects. Thus, remnant ejecta from the BALR may be the cause of associated complex absorption systems. One speculation is that the mass swept up in the outflow may become more diluted with hydrogen, explaining why the BALR appears metal enriched, while the clouds causing the associated complexes of absorption do not. If this is the case, either a radio structure becomes visible at about the same time BALs dissipate, or when viewed from other aspect angles that are out of the plane of the BALR disk, both the radio structure and the 
complex absorption are visible. This latter possibility is more consistent with a picture in which QSOs have steady-state BALRs. One can not rule out that freefree absorption in the BALR causes the radio structure to be absorbed. If such a speculation holds, the implication is that the BALR is capable of affecting regions surrounding a QSO in excess of a few $100 \mathrm{kpc}$.

\section{Summary of Implications}

In section 4, I discussed constraints on the global and local values of the BALR covering factor. The observations of BAL QSO EL profiles and residual intensities in BAL troughs necessitates a small global covering factor; however, in order to have a viable model for the acceleration of BALR clouds, a large local covering factor is required. I discussed why a high ionization broad EL component may arise in the BALR; the lack of broad [OIII] $\lambda 5007$ emission in QSO spectra then requires that electron densities in the BALR be less than $10^{6}-10^{8} \mathrm{~cm}^{-3}$. These densities generally imply upper limits on the distance to the BALR of a $100-1000$ pc. The constraint that the BALR lies beyond the broad Ly- $\alpha$ emitting region and the recent observations which show no change in the velocity of individual $B A L$ components put a lower limit on the distance to the BALR of $1-7 \mathrm{pc}$. All of these constraints, coupled with the need to have only symmetric emission components arising from the BALR, strongly indicate a disk-like distribution for the BALR.

Column density determinations for BAL clouds show levels of ionization that are difficult to understand using solar abundances and simple models for the ionization. In particular, however, the observation that the $\mathrm{H}^{0}$ column density can be only a factor of 2 or so greater than the $\mathrm{C}^{+}$column density in a specific absorption component suggests BALR abundances which are very enhanced (10 - 100 times) relative to solar values. Observations of iron emission in BAL QSOs may also be telling us that abundances are enhanced.

The further observation that many radio loud QSOs have associated complexes of absorption that are not quite extensive enough to have the QSO placed in the radio quiet $B A L$ class suggests that the BALR may affect the region surrounding a QSO out to at least several hundred kpc. If this were the case, some connection between the observed presence of radio emission and either the viewing angle needed to observe BALs or the evolutionary state of the BALR must exist.

Collectively, the observations and constraints which have been discussed offer a snapshot of what the BALR may look like. The notion that it is a high ionization component of a QSO's broad ELR is probably correct, but the source of the BALR clouds need not be the same as the other clouds giving rise to a QSO's ELs. Indeed, the properties of the clouds producing the broad Ly- $\alpha$ emission, BALs and the narrow ELs are very different in terms of the constraints which I have discussed. In particular, the clouds producing the broad $\mathrm{Ly}-\alpha$ emission are known not to be able to occult the continuum source either individually or collectively, which is in contrast to the BALR clouds. The symmetric profiles that are characteristic of the $\mathrm{Ly}-\alpha$ emission may be an indication that rotation is important rather than outflow in the inner, denser, lower ionization broad ELR. Also, as the observations show, the BALR must be incapable of producing Ly- $\alpha$ emission. As far as other broad ELs are concerned, the extent to which the BALR contributes to the emission is unclear. Certainly, there is a very efficient RLS mechanism for producing NV in the BALR and collisional excitation may be important to varing degrees. As discussed by Bev Wills and Alexei Fillippenko at this symposium, all of these different ELRs may blend into one another to some degree. 


\section{Problems and Future Work}

One of the main problems in this area is the lack of a suitable model for the BALR level of ionization. Until a reliable model is found, many of these results must be evaluated carefully. Of course, the situation is complicated because deciding on the ionization mechanism is only part of the problem; the other part is adopting the correct abundances. If models rely on energy input into the BALR clouds via central source photoionization, they should try to properly take into account attenuation of the Lyman continuum by any BAL resonance line transitions that may be located in the Lyman continuum. The most straightforward way to do this will be to wait for UV observations in the Lyman continuum of BAL QSOs. This will not only tell us how the Lyman continuum is chopped-up by the BALs, but it will also give us new and important information on the level of ionization in the BAL clouds. One starting point for considering models for the level of ionization would be to try to produce a region where the neutral hydrogen density is not too much greater that the singly ionized carbon density. However, one should keep in mind that low ionization components are not typical of BALR clouds. Other important theoretical work needs to be done in the areas of acceleration of the clouds and the actual source of the clouds. Two possible sources for the clouds are the region which gives rise to the inner and denser Ly- $\alpha$ emitting region and supernovae of massive stars.

Important observational work is needed in the areas of detailed studies of individual BAL QSOs and on the statistics of the EL and BAL properties of QSOs. In particular, placing limits on possible changes in BAL profiles with time, placing limits on the amount of broad [OIII] emission present in a QSO's spectrum, the derivation of BALR column densities and detailed analysis of the Ly- $\alpha / \mathrm{NV}$ region in BAL QSO spectra for the purpose of studing RLS, covering factor, aspect angle and radiative transfer effects are all very necessary. As studies of polarization and optical variability properties are sparse, more work in these areas is needed. The statistical questions fit into two catagories. First, there is the question of whether there are changes in the incidence of BAL QSOs as a function of redshift or luminosity. Second, there is the question of how the EL properties of BAL QSOs compare with non-BAL QSOs within a specific redshift range and luminosity interval. Such a study should consider the absorption and EL properties of steep and flat spectrum radio sources as well. To answer both of these questions, one would need samples in which the selection criteria as a function of absorption and emission line properties are well understood. Results dealing with the second question would tell us something about aspect angle effects and the value of the BALR covering factor as a function of QSO EL properties. Of course, in the simplest case where there is a spherically symmetric geometry and the BALR covering factor is a constant in all QSOs, one expects the distribution of EL properties in BAL QSOs to be identical to the distribution of EL properties in non-BAL QSOs. As was the point of most of the discussion presented here, this possibility is almost certainly ruled out. The observations now challenge us to put together a self-consistent snapshot of the distribution of QSO EL and BAL properties as a function of geometry, aspect angle and radiative transfer effects.

\section{Acknowledgements}

My work on BAL QSOs has benefited from discussions with Frank Briggs, Craig Foltz, Cyril Hazard and Art Wolfe; I would especially like to acknowledge continuing discussions with Ray Weymann on this subject. 


\section{References}

Briggs, F.H., Turnshek, D.A., and Wolfe, A.M. 1984,Ap.J.(Letters), 287, 549.

Canfield, R.C., and Puetter, R.C. 1981, Ap.J., 226, 1.

Drew, J., and Gidding, J. 1982, M.N.R.A.S., 201, 27.

Drew, J., and Boksenberg, A. 1984, M.N.R.A.S., $211,813$.

Foltz, C.B., Wilkes, B.J., Weymann, R.J., and Turnshek, D.A. 1983a, P.A.S.P., 95, 341.

Foltz, C.B., Weymann, R.J., Hazard, C., and Turnshek, D.A. 1983b, P.A.S.P., 95, 117.

Foltz, C.B., Weymann, R.J., Morris, S.L., and Turnshek, D.A. 1986a, in preparation.

Foltz, C.B., Weymann, R.J., Peterson, B.M., Sun, L., Malkan, M.A., and Chaffee, Jr., F.H. 1986b, Ap.J., submitted.

Gaskell, C.M. 1982,Ap.J., 263, 79.

Hartig, G.F., and Baldwin, J.A. 1986, Ap.J., in press.

Hazard, C., Morton, D.C., Terlevich, R., and McMahon, R.G. 1984, Ap.J., 282, 33. Junkkarinen, V.T. 1983, Ap.J., 265, 73.

Morris, S.L., Weymann, R.J., Foltz, C.B., Turnshek, D.A., Schectman, S., Price, C., and Boroson, T.A. 1986, Ap.J., submitted.

Scott, J.S., Christiansen, W.A., and Weymann, R.J. 1984, unpublished preprint.

Stocke, J.T., Foltz, C.B., Weymann, R.J., and Christiansen, W.A. 1984, Ap.J., 280, 476.

Turnshek, D.A., Weymann, R.J., Liebert, J.W., Williams, R.E., and Strittmatter, P.A. 1980, Ap.J., 238, 488.

Turnshek, D.A. 1981, Ph.D. Thesis, University of Arizona, Univ. Microfilm Inc., Ann Arbor, Michigan.

Turnshek, D.A. 1984a, Ap.J., 280, 51.

Turnshek, D.A. 1984b, Ap.J.(Letters), 278, L87.

Turnshek, D.A., Foltz, C.B., Weymann, R.J., Lupie, O.L., McMahon, R.G., and Peterson, B.M. 1985, Ap.J.(Letters), L1.

Turnshek, D.A., Foltz, C.B., and Weymann, R.J. 1986, preprint.

Turnshek, D.A., and Grillmair, C. 1986, in preparation.

Turnshek, D.A., Briggs, F.H., Foltz, C.B., and Weymann, R.J. 1986, in preparation.

Wampler, J.S. 1986, preprint.

Weymann, R.J., Williams, R.E., Peterson, B.M., and Turnshek, D.A. 1979, Ap.J., 234, 33.

Weymann, R.J., Scott, J.S., Schiano, A.V.R., and Christiansen, W.A. 1982, Ap.J., 262, 497.

Weymann, R.J., and Foltz, C.B. 1983, Proc. of the 24th Liege Symposium: Quasars and Gravitational Lenses.

Weymann, R.J., Turnshek, D.A., and Christiansen, W.A. 1985, Astrophysics of Active Galaxies and Quasi-Stellar Objects, ed. Miller, J.S.

Wilkes, B.J., and Carswell, R.F. 1982, M.N.R.A.S., 201, 645.

Wilkes, B.J. 1985, Ap.J.(Letters), 288, L1.

Wolfe, A.M., Turnshek, D.A., Smith, H.E., and Cohen, R. 1986, Ap.J.(Supplement), in press. 
DISCUSSION

Blandford : If, as you suggest, reasonance line scattering and anisotropic distribution of the absorbing material are both important features of BAL Quasars, then you expect to see some substantial polarization changes through the line profiles and this might give a unique diagnostic for the cloud distribution and optical depths. What prospects are there for doing high resolution spectropolarimetry on these objects?

Turnshek : Under certain circumstances, you would indeed expect to see a polarization signature of some sort. However, if you had multiple scatterings, this would cause depolarization and the prospects for detecting anything would be poor. I have done resonance line scattering polarization calculations for the case of a disk geometry. The calculations show that for the case where you have photons typically scattered once, you might expect the resonance line scattered part of an emission line to be polarized by as much as 20-25\%. From the observation you could determine the plane of the disk on the sky. For the case where BALs are seen (i.e. your viewing angle passing through the plane of the disk) you would have maximum polarization. As your line of sight started to miss the disk (i.e. viewing angles out of the plane) you would not expect to see BALs and you would get an interesting "W" shaped polarization profile as a function of wavelength. At polar angles there would be no polarization. The observation would not be trivial because the continuum of BAL QSOs in sometimes polarized. In anycase, you would have to subtract this continuum contribution off. There might also be an unpolarised part to the emission line. The prospects are not good for detecting this with instruments that exist today. It may be that space Telescope offers the best hope of detecting this because integration times would be long and night sky polarization from the ground could cause a problem. However, it would be good to get an expert opinion in this area.

Burbidge : I have two questions: First, you suggested that from the absorption lines you could derive heavy element abundances $\sim 100$ times solar. If the absorption and emission arises in the same (contiguous) region, how do you reconcile this with the fairly normal (solar) abundances deduced from the emission lines ? Second, if the broad absorption line QSOs are part of the normal QSO population should you not see the same incidence of sharp absorption features in them that you see in the non BAL QSOs in the same emission-line redshift range ?

Turnshek : For the first question, my answer is that in the case of BALs I believe you can more accurately determine column densities as long as you do not have unocculted emission at the wavelength of the absorption features. In the case I have discussed, you have a fairly isolated absorption component at $14,100 \mathrm{~km} \mathrm{~s}^{-1}$. The problem with abundance determinations from emission lines is that there is a collection of clouds which, as we have heard at this meeting, have a wide range of properties in terms of densities and levels of ionization. There is kinematic evidence, 
and evidence from BAL QSOs as well, that different emission lines can come from different regions or clouds. Therefore, I would argue that you need a very good model to properly constrain abundances from emission line observations.

For the second question, my answer is that BAL QSOs are necessarily excluded from spectroscopic surveys which try to determine the distribution and statistics of narrow, isolated absorption lines. This is because you can not adequately assess the path length covered by the survey and because there could be contamination from narrow components which make up the BALs. On the other hand, I do know of some BAL QSOS which have narrow absorption lines and these lines are consistent with the properties of the so-called intervening population of absorption lines. Of course, you would expect this.

Malkan : To agree with your statement that the BAL phenomenon is independent of $\mathrm{z}$ : of the $<100$ PG quasars with $\mathrm{z}<1.5$, we now know that at least three are BAL QSO's. The newest one, found by IUE observations, has a redshift of 0.089 , with strong, relativity narrow NV and CIV troughs.

Turnshek : That is very interesting, Of course, there is still the question of the fraction of QSOs with BALs at the highest redshifts, $z>3$.

Wampler : Harting and Baldwin (1986) have pointed out that the spectra of BAL quasars are different from non-BAL quasars. The region to the red of about $\lambda_{0} \sim 2000 \mathrm{~A}$ is generally free of broad absorption lines and the corresponding regions can be compared. They find that BAL quasars have much stronger FeII emission than non-BAL quasars. This strongly suggests that many quasars are not BAL quasars.

Turnshek : Yes, this is true and very important. However, it is not completely clear what the statistics are yet. If there are some BAL QSOs which have weak FeII emission, these objects may just have systematically smaller BALR covering factors. Of course, it is also possible that emission line properties are aspect angle dependent. If the BALR is confined to a disk, emission line properties may be diffrerent when viewed in the plane of the disk.

Wilkes : Geoff Burbidge expressed concern with the discrepant abundances between BALR clouds and BELR clouds especially if they are the same clouds. I understood you to say that BALR clouds contribute to the broad emission but not that they are the BELR clouds. Is this correct?

Turnshek : Yes. The BAL clouds certainly have some very different properties from the "standard" BEL clouds. For one thing the BAL clouds are capable, at least collectively, of completely covering a region the size of the inner $L \alpha$ emitting region, which is probably $>10^{18} \mathrm{~cm}$. However, the clouds giving rise to this inner $L \alpha$ emitting region are not even collectively capable of covering a region which is probably $10^{14} \mathrm{~cm}$. It may be necessary to have these two types of clouds originating from two completely different sources. For example, the inner $L \alpha$ emitting clouds may come from the accretion disk, but the BAL clouds may come from supermassive stars. 